\title{
Physician choices in pulmonary embolism testing
}

\author{
Sahar Zarabi BSc, Teresa M. Chan MD MHPE, Mathew Mercuri PhD, Clive Kearon MD PhD, * \\ Michelle Turcotte MSc, Emily Grusko MD, David Barbic MD MSc, Catherine Varner MD MSc, \\ Eileen Bridges MD BSc, Reaves Houston BSc, Debra Eagles MD MSc, Kerstin de Wit MBChB MD MSc
}

Cite as: CMAJ 2021 January 11;193:E38-46. doi: 10.1503/cmaj.201639

\begin{abstract}
BACKGROUND: Evidence-based guidelines advise excluding pulmonary embolism (PE) diagnosis using D-dimer in patients with a lower probability of PE. Emergency physicians frequently order computed tomography (CT) pulmonary angiography without D-dimer testing or when D-dimer is negative, which exposes patients to more risk than benefit. Our objective was to develop a conceptual framework explaining emergency physicians' test choices for PE.
\end{abstract}

METHODS: We conducted a qualitative study using in-depth interviews of emergency physicians in Canada. A nonmedical researcher conducted in-person interviews. Participants described how they would test simulated patients with symptoms of possible PE, answered a knowledge test and were interviewed on barriers to using evidence-based PE tests.

RESULTS: We interviewed 63 emergency physicians from 9 hospitals in 5 cities, across 3 provinces. We identified 8 domains: anxiety with $\mathrm{PE}$, barriers to using the evidence (time, knowledge and patient), divergent views on evidence-based PE testing, inherent Wells score problems, the drive to obtain $\mathrm{CT}$ rather than to diagnose $\mathrm{PE}$, gestalt estimation artificially inflating PE probability, subjective reasoning and cognitive biases supporting deviation from evidence-based tests and use of evidence-based testing to rule out $P E$ in patients who are very unlikely to have PE. Choices for PE testing were influenced by the disease, environment, test qualities, physician and probability of PE.

INTERPRETATION: Analysis of structured interviews with emergency physicians provided a conceptual framework to explain how these physicians use tests for suspected PE. The data suggest 8 domains to address when implementing an evidence-based protocol to investigate PE.
$\mathbf{P}$ ulmonary embolism (PE) occurs when a blood clot lodges in the pulmonary arteries. If left untreated, the disorder can progress, causing worsening morbidity and may become fatal. ${ }^{1}$ Because of the acute nature of this condition, many patients with PE present to the emergency department.

Diagnosing and excluding PE using computed tomography pulmonary angiography (CTPA) alone can be problematic because of radiation exposure, anaphylaxis to contrast, misdiagnosis and "overdiagnosis" of inconsequential PE' (leading to unnecessary anticoagulation therapy and psychological distress $\left.{ }^{3}\right)$. Choosing Wisely $y^{4,5}$ and the guideline from the American College of Physicians ${ }^{6}$ recommend the use of risk stratification tools, including the Pulmonary Embolism Rule-out Criteria (PERC) clinical decision rule, ${ }^{7}$ the Wells score ${ }^{8}$ and blood concentration of D-dimer. These tools use different predetermined diagnostic algorithms to indicate the need for CTPA. ${ }^{8-11}$ Evidencebased guidelines discourage further testing in patients at lower risk who have normal D-dimer levels, where imaging can cause more harm than benefit. ${ }^{12,13}$ However, many emergency physicians opt for CTPA as a stand-alone test for PE. ${ }^{14-17}$

It remains unclear why emergency physicians sometimes do not use validated diagnostic PE tools. Furthermore, implementation of computerized decision support systems has had little success in modifying this behaviour. ${ }^{18,19}$ We sought to develop a conceptual framework to describe how Canadian emergency physicians test for PE, and to document the cognitive and contextual barriers to using existing evidence-based diagnostic PE pathways.

\section{Methods}

\section{Study design and population}

This was a qualitative, 4-part interview study. We followed the COnsolidated Criteria for REporting Qualitative Research (COREQ) guideline for reporting. Participants were staff emergency physicians in 
Canada who were invited to an interview on "clinical decisionmaking." We interviewed physicians from 3 hospitals in Hamilton to develop a provisional framework describing how emergency physicians test for PE, and why they choose the tests they use. We were mindful that Hamilton is the birthplace of Canadian thrombosis medicine, so we then interviewed emergency physicians from sites across Canada, until thematic sufficiency had been reached. The participating hospitals did not have computerized decision support for PE testing.

We sent an email invitation to every staff emergency physician working in the participating hospitals (9 hospitals in Hamilton, Ottawa, Montréal, Vancouver and Toronto). We used snowball sampling when there was an insufficient response to the email invitation.

\section{Interviews}

The structured interview was designed as a cognitive task analysis, a technique which elicits practitioners' thinking around parts of their work that require thought, planning and action. The interview was piloted and refined before implementation. Appendix 1 (available at www.cmaj.ca/lookup/doi/10.1503/ cmaj.201639/tab-related-content) includes the interview, links to the videos, an example mind map and the questionnaire. In phase 1 of the interview, the participant recalled a patient they tested for PE and explained their diagnostic approach and reasoning. In phase 2 , the participant watched 2 videos of patients with possible PE symptoms in a simulation (the videos were chosen randomly from a selection of 4 videos). Participants were then asked to explain to the interviewer how they would test the patient (step by step) by drawing a mind map. In phase 3, they completed a questionnaire on their knowledge of the Wells score, ${ }^{8}$ age-adjusted D-dimer ${ }^{9}$ and the PERC rule. ${ }^{7}$ The interview finished with open-ended questions about barriers to using the diagnostic guidelines for PE (phase 4). All interviews were identical with the exception that participants from Hamilton reviewed an additional video of a patient with possible deep vein thrombosis. Our study analyzed only the PE-related cases. The interviews were conducted at each hospital in a private room. Our investigators trained the site research staff who did not have previous qualitative research experience and were either research assistants or undergraduate students with no previous relation to the participants. Interviews were audiorecorded and later transcribed.

\section{Qualitative analysis}

We used a constructivist grounded theory technique ${ }^{20}$ to develop a conceptual model that explained how emergency physicians choose PE testing. The qualitative analysis was performed by an investigatory team comprising an emergency physician researcher specializing in knowledge translation and education (T.M.C), an emergency and thrombosis physician who specializes in diagnostic PE research (K.d.W), a nonphysician expert in physician practice variation research (M.M.) and an undergraduate student with no previous experience of PE (S.Z.). The investigatory team met to discuss their inherent stances and assumptions to ensure reflexivity before the start of the study.
For the interviews involving participants in Hamilton, each interview was coded separately by 3 researchers (K.d.W., T.M.C. and M.M.) using a constant comparative approach. ${ }^{21}$ An iterative process was used whereby assigned codes were reviewed by the research team, who developed and refined a common code book. The team met on 7 occasions. We assigned similar codes to common themes to create our initial framework. The interviews were analyzed while interviews were ongoing. We ceased the interviews when no new themes emerged.

To refine this initial framework, 2 investigators (S.Z. and K.d.W.) coded the transcripts and mind maps from interviews conducted in 6 additional emergency departments, using the previous framework codes. The researchers met a total of 6 times. Where the investigators were unable to identify a suitable code, they agreed on new codes that were mapped to preexisting themes or a new theme was identified. Themes were grouped into domains for the final version of the framework.

\section{Statistical analysis}

For each clinical case discussed in the interviews, 2 researchers independently recorded which clinical decision rules were used for PE testing. Participant demographics and quantitative interview data were reported as medians and interquartile ranges (IQRs) or proportions.

\section{Ethics approval}

Approvals were obtained from the Research ethics boards for all participating hospitals.

\section{Results}

We conducted 63 interviews (participant demographics found in Table 1). Between 2015 and 2016, we interviewed 16 emergency physicians working at 1 of 3 hospitals in Hamilton, from which 6 themes were derived (Appendix 2, available at www.cmaj.ca/ lookup/doi/10.1503/cmaj.201639/tab-related-content). An additional 47 physicians completed interviews in the refinement stage, which took place between 2017 and 2018. Our analysis yielded 7 new themes and the final framework included 8 domains, which are described in the following paragraphs (Table 2). Example quotes can be found in Table 3.

\section{Anxiety with pulmonary embolism}

Participants viewed PE as a fatal disorder and indicated that identifying dangerous diagnoses is a pivotal role for emergency physicians. Missing the diagnosis of PE implied failing in this role. Therefore, the decision to start or not to start testing for PE evoked anxiety. Some participants expressed anxiety about using D-dimer or decision rules because of a lack of confidence in their safety or because of tool complexity and the potential for error.

\section{Barriers to using the evidence}

The time of day and degree of crowding in the emergency department affected the use of evidence-based tools, especially D-dimer, which was cited as delaying the ordering of an inevitable CTPA. 
Table 1: Participant demographic characteristics

\begin{tabular}{lc} 
Characteristic & $\begin{array}{c}\text { No. (\%) of } \\
\text { participants } \\
n=63\end{array}$ \\
$\begin{array}{l}\text { Years since medical degree was obtained, } \\
\text { median (IQR) }\end{array}$ & $12(7-25)$ \\
Years of EM residency training, median (IQR) & $2(1-5)$ \\
\hline Training route & \\
\hline FRCPC (EM) & $25(40)$ \\
\hline CCFP-EM & $36(57)$ \\
\hline ABEM & $1(2)$ \\
\hline Practice entry & $1(2)$ \\
\hline Type of emergency department & \\
\hline Academic & $50(79)$ \\
\hline Community & $9(14)$ \\
\hline Both & $4(6)$ \\
\hline Nearest city & \\
\hline Hamilton & $16(25)$ \\
\hline Toronto & $15(24)$ \\
\hline Ottawa & $10(16)$ \\
\hline Montréal & $11(17)$ \\
\hline Vancouver & $11(17)$ \\
Note: ABEM = American Board of Emergency Medicine, CCFP $=$ Canadian College of \\
Physicians of Canada, IQR = interquartile range. \\
*Unless specified otherwise.
\end{tabular}

Eight participants did not know that the Wells score and D-dimer were validated PE tests. Some participants felt patients attended the emergency department with an expectation of CT scanning.

\section{Divergent views on evidence-based pulmonary embolism testing}

Participants either liked using evidence-based diagnostic PE tools or expressed a range of negative opinions about these tools. The views were often contradictory: for example, PE clinical decision rules were cited as both saving time and taking too long to use, reducing testing and causing unnecessary testing, helping with patient flow through the emergency department and hindering patient flow.

\section{Inherent problems with the Wells score}

The Wells score was singled out by participants as being problematic to use in the emergency department. Many disliked that the Wells score is an intermediary step in the diagnostic process (leading to D-dimer testing or CT). Several disliked the subjective allocation of points for "PE being the most likely diagnosis," and participants would allocate these points liberally, based on the presence of PE risk factors rather than on the likelihood of there being an alternative cause for the symptoms. A recurring concern was that the score added to an emergency physician's cognitive load. Several found it frustrating that there are many new ways to interpret this score.
Pulmonary embolism testing must include $\mathrm{CT}$ imaging In many instances, the participant saw CTPA as inevitable when there was suspicion of PE. For some, deciding to test for PE was synonymous with ordering CTPA. Computed tomography was considered the most trusted test for PE by the participants. Computed tomography pulmonary angiography was frequently referred to as the "definitive test;" obtaining a CTPA removed the anxiety of missing PE, the anxiety around deciding whether to test for PE and the anxiety associated with trusting D-dimer and a clinical decision rule. The goal was to obtain CTPA rather than diagnose PE. By arranging a CT, they handed over responsibility for PE testing to the radiologist. No participant remarked on the possibility of false-positive or false-negative $\mathrm{CT}$ results. The CTPA report was seen as the radiologist's domain and the CT results accepted without question.

\section{Gestalt artificially inflates pulmonary embolism pretest probability}

One of the strongest themes was working with gestalt. Several participants were so comfortable with their gestalt that they would trust gestalt over clinical decision rules, D-dimer level and imaging results (e.g., by ordering CTPA for a patient with a low Wells score and normal level of D-dimer). We observed that when a participant used gestalt, they commonly overestimated the pretest probability of PE. Use of gestalt frequently led to ordering CTPA. Gestalt was mostly stated as "high risk" (when the Wells score was low). High risk could refer to the patient having risk factors for developing PE (e.g., being treated for cancer or having a history of PE). The word "risk" was transferred from one term (risk factor) to another (high risk) without further thought. The term high risk was also used when participants felt anxious about PE. Other times risk referred to the potential for a hemodynamically unstable patient to die. Risk signalled low confidence in D-dimer to safely rule out PE or that D-dimer would be a nuisance rather than a help. Labelling the patient as high risk facilitated obtaining CT.

\section{Subjective reasoning and cognitive bias}

Almost all participants stated they would follow an evidencebased strategy. However, in some instances, the diagnostic process did not include clinical probability stratification or D-dimer, and the participant defaulted to ordering CT. Physician reasoning included the following: they believed (wrongly) that the patient was an exception to the use of the Wells score; the Wells score often differed from their own gestalt estimate of pretest probability; they guessed that the Wells score would be high or that D-dimer would be elevated; and they appeared to "inflate" the Wells score to avoid the use of D-dimer.

\section{Clinical decision rules are used mainly to rule out pulmonary embolism}

Evidence-based testing for PE appeared more likely to occur when the participant's gestalt pretest probability of PE was low. Participants appreciated being able to document the PERC rule in this instance for medicolegal reasons.

We were unable to detect theme patterns according to training, years in practice, city, and academic versus community practice. 


\section{Domain}

Anxiety with PE

Barriers to using the evidence

Divergent views on evidence-based PE testing

Inherent Wells score problems

Theme

Subtheme

$\mathrm{PE}$ is a dangerous diagnosis

PE triggers anxiety for physicians

Time pressure

Knowledge

Patient influence

Clinical decision rules are useful

Skepticism about clinical decision rules

$\mathrm{CT}$ is an end point
Focus on chest pain

Diagnostic testing focuses on dangerous diagnoses Physicians should avoid premature diagnoses Reluctance to think there may be no diagnosis Common use of "rule out" rather than "rule in" Lack of comfort with undiagnosed chest pain without PE testing

Diagnosing PE is a complex process compared with other conditions Difficulty differentiating between no and low probability of PE Clinical decision rules do not determine the trigger to test for $\mathrm{PE}$ If the patient has any risk factor for PE they must be tested for PE Testing because the patient is unstable

Testing because patient is hypoxic

Testing because there is no other diagnosis

Testing because patient is tachycardic

Time of the day influences choice to follow evidence-based testing Evidence-based medicine is bad for patient flow Time of day influences choice of test Do not use D-dimer if this is a busy shift D-Dimer takes too long to use in the diagnostic process Gestalt is faster than using a clinical decision rule Clinical decision rules take too long to use Clinical decision rules add to cognitive overload

PE clinical decision rules have not been validated Physician does not know how to use PE clinical decision rules Frustration when Wells score and D-dimer do not match Ultrasonography of the legs is part of the PE testing algorithm

Sometimes the patient is particularly concerned about PE Patients generally expect imaging

Clinical decision rules help with flow Clinical decision rules are time saving

Clinical decision rules reduce $\mathrm{CT}$ Clinical decision rules reduce imaging Clinical decision rules are validated Confidence in evidence-based medicine Faster to rule out $\mathrm{PE}$ with clinical decision rules If it seems PE is the most likely diagnosis then use a clinical decision rule

Clinical decision rules take too long to use

Clinical decision rules are aids only

Clinical decision rules lead to unnecessary tests

Clinical decision rules are teaching tools only

Lack of confidence in clinical decision rules

D-Dimer is for other specialists

No belief in evidence-based medicine

Physician says they use evidence, but in practice they do not

The Wells score is an intermediate step, not an end point in the process Interpretation of the Wells score keeps changing - this is confusing Still using 3-tier Wells despite more recent evidence for 2-tier score Wells scoring is a dynamic process as you work a patient up Physicians allocate 3 points for "PE most likely" if there are any PE risk factors Frustration when clinical decision rules contradict gestalt

Frustration with subjective allocation of PE most likely in the Wells score Clinical decision rules add to cognitive overload

CT scanning is inevitable when testing for PE

If a physician has any suspicion of $\mathrm{PE}$, the patient will have a CT

If a patient has PE risk factors they need diagnostic imaging for PE Overriding confidence in diagnostic imaging

Imaging overrides clinical decision rules Computed tomography is easily accessible

Thrombosis physicians support imaging if there is any doubt Ventilation/perfusion scanning is not easily accessible

Computed tomography is useful for older patients who might have other pathology Imaging can be conducted the next day if after hours

If the patient is unstable, they need PE diagnostic imaging 


\section{Domain}

Theme

Gestalt inflates PE

probability

Subjective reasoning and cognitive bias

Clinical decision rules are used mainly to rule out PE

\author{
Overriding confidence in own gestalt \\ Gestalt overrides clinical decision rules \\ Gestalt overrides evidence-based medicine \\ Gestalt overrides diagnostic imaging \\ Using gestalt leads to PE testing, high pretest probability estimate and allocation of PE most \\ likely 3 points on the Wells score \\ Any PE risk factor leads to high-risk gestalt estimate \\ $\mathrm{PE}$ is most likely diagnosis leads to high-risk gestalt estimate \\ If gestalt for PE is low, then physician will order D-dimer \\ Physician considers using evidence-based testing but decides not to \\ Guessing that the D-dimer will be elevated \\ Guessing the Wells score, not calculating it \\ Inflating the Wells score \\ Thinks this patient is an exception to using the Wells score \\ Skips steps in the evidence-based algorithm \\ Clinical decision rules justify the decision to discharge a patient \\ Confidence that negative D-dimer excludes PE \\ Confident communication about clinical decision rules with patients \\ Clinical decision rules help address medicolegal anxiety \\ Documentation of a rule gives legal support for not doing a CT \\ If your gestalt for $P E$ is low, use a clinical decision rule \\ Pulmonary Embolism Rule-out Criteria (PERC) supports not testing for PE when you do not \\ think you have to test \\ A negative PERC score is an end point for PE testing
}

Note: $\mathrm{CT}=$ computed tomography, $\mathrm{PE}=$ pulmonary embolism

\section{Table 3 (part 1 of 3): Quotes illustrating the framework themes and subthemes}

\section{Anxiety with PE}

PE triggers anxiety for physicians

$\mathrm{PE}$ is a dangerous diagnosis

\section{Barriers to using the evidence}

Time pressure

Knowledge

Patient influence
I don't think you would call it a barrier, a challenge, is um so kind of like suspicion... suspicion creeps. We talk about PE a lot in the emergency department ... (ID16) Fear. I think that many physicians are afraid of the negative impact of missing a pulmonary embolism. (ID42)

PE is kind of bad because you know as the risk of being, you know, life threatening and fatal including in younger people, its signs and symptoms of it can really blend with a lot of other presentations including benign things and third, its relatively common so all those things together can kind of be the bogey man of emergency department diagnosis right? (ID4)

In the emergency department, when you hear hoofbeats, you're supposed to think of 2 things. The first thing is zebras, which are rare, off the wall things, because if you don't think about it, no one else will, and 2, are hippos. Big lumbering animals which if you miss them will crush you to death, or in this case the patient. (ID37)

When it's low risk they suggest getting a D-dimer first and then get a follow-up testing. Um ... which can be, which unnecessarily increases the time the patient is in the department. (ID16)

Stuck out in my mind in the sense that she was you know essentially zero on the Wells score and ... she had a PE. (ID4)

You only need one positive risk and then that automatically dumps you into high or moderate risk category. (ID 62)

If you're already going down the hole of, like the rabbit hole of the patient insisting on a test, the rules aren't, the rules no longer apply. (ID26)

Skip the step of the clinical decision rule and order what they want because with the patient in front of you, you are worried about them and it doesn't help that they were Wells score negative and they went home and died from a PE so sometimes we have to skip it. (ID3) 


\section{Divergent views on evidence-based PE testing}

Clinical decision rules are useful

Skepticism about clinical decision rules

Inherent Wells score problems

\section{PE testing must include $\mathrm{CT}$}

$\mathrm{CT}$ is an end point

Gestalt drives testing and inflates PE probability

One of the most important scoring factors is your clinical judgment. So, I don't have to look at a scoring system to know that my clinician judgment is still going to be up to me. (ID27) I think for some of us even if the rule were to score them in a certain category, if our clinical gestalt tells us differently based on potentially a case we've seen before ... and you know this feeling like it's gonna nag us forever if we don't get it. (ID34)

On the physical exam I'm gonna look to see if he has any swelling in his calves or anything going ... but even if he doesn't ... he's at high risk, we're gonna have to go after that, and the only way to go after that is a CT PE study, which we can get done. (ID22)

I don't find that rules are going to be overly helpful if you're clinical gestalt from talking to someone is sufficient enough to have you concerned to order a study. For me personally, whether or not her Wells score is 2 or 5 is not gonna change how I actually work up. (ID12) I wouldn't, umm, wouldn't use Wells, I would just go straight to imaging, so CT chest just because his risk is much higher, umm and then investigation is done. (ID23)

If your clinical suspicion thinks that PE is likely, then that trumps all of that scores. (ID13) I guess one of the challenges with clinical decision rules is sometimes you really want to do something although technically they don't fit in the clinical decision rule so you will have to wrestle with yourself. Are you going to let your clinical decisions trump the decision rule? (ID21) 


\section{Subjective reasoning and cognitive bias}

The answer is actually he is excluded. He is overtly excluded [from having the Wells score applied]. He's had a previous PE which is a point, but he has a known disease like is Factor $V$ Leiden. He is just out, like he is completely out on all of those derivation studies, he is not even included. (ID22)

You know your mother, grandmother didn't look at a recipe card to make your favourite dishes by memory right? She gave it to you and you wrote it down, you do it a few times and you do it often enough, you stop looking at the recipe card. You'd also tinker with the recipe card to add a little butter or mint or something, you tinker with it a little bit to make it suit you better. (ID38)

Would probably be doing the D-dimer just for completion's sake to say that I've done it, sometimes I just kinda, it just shows that it's being done, um, but you know, I've already made the decision that I'm going to need to do a CT. (ID11)

"t wasn't a tough decision. It wouldn't matter what the Wells criteria said I would have gone to it [CT] anyways. (ID27)

\section{Clinical decision rules are used mainly to rule out PE}

So, I suspect that I'm far more likely to use them [clinical decision rules] when in the back of my head I say oh yeah, for sure this patient is PERC negative so I'm gonna put it on the chart. (ID12)

If you think it's like low probability (not no probability), and you, just before you [discharge them] saying this isn't anything, you have to be negative for the PERC rule. (ID63)

Note: $\mathrm{CT}$ = computed tomography, $\mathrm{PE}=$ pulmonary embolism, $\mathrm{PERC}=$ Pulmonary Embolism Rule-out Criteria.

Table 4: Participant use and knowledge of decision rules

\section{Risk stratification tool used in each case}

PERC Wells

Wells

PERC gestalt

Gestalt

\section{Knowledge test results}

Correctly listed PERC items

Correctly listed Wells items

Correctly described age-adjusted D-dimer

Note: PERC $=$ Pulmonary Embolism Rule-out Criteria.

Diverse opinions about using evidence-based PE testing were voiced by all groups. Table 4 summarizes the PE testing and knowledge test results.

\section{Interpretation}

Our qualitative exploration of how Canadian emergency physicians test for PE yielded important insights. We found physicians felt anxious about missing PE, knowing when to test for PE and using evidence-based PE tests. Physician knowledge, time pressure and patient expectations were barriers to using evidencebased testing, with divergent opinions about the evidence. We found practical problems with using the Wells score in the emergency department. There was a focus on obtaining CTPA rather than diagnosing PE. Together, these issues explained the preference for using gestalt estimation of pretest probability, which appeared to artificially inflate the probability of PE. Physicians used several cognitive strategies to perpetually choose CTPA over evidence-based testing, which was more often reserved for patients who were very unlikely to have PE.

Gestalt was used in $66 \%$ of the cases and was the most common subjective reasoning tool to justify ordering CTPA over D-dimer. A study involving consecutive patients presenting to the emergency department in France and Belgium found that a physician gestalt estimate of pretest probability performed similarly to the Wells score. ${ }^{22}$ We found physician gestalt was used in place of a clinical probability score; however, in our study gestalt was not a pretest probability gauge. Instead, participant gestalt statements signalled the need or urgency for CTPA. Stating a patient was at high risk for PE permitted the physician to bypass D-dimer or ignore a normal D-dimer result. Gestalt is a "workaround" in the era of clinical probability estimation. Evidence-based PE testing has changed physician vocabulary. At first glance, physicians appear to follow evidence-based guidelines, but in reality, this evidence has little impact on the choice of test.

Our findings concord with previous research. There are only 2 previous studies exploring emergency physician behaviour around PE testing. ${ }^{23,24}$ In addition to finding a reliance on gestalt, both studies also reported contrasting views on the value of evidence-based PE testing and noted that physician gestalt tended to exaggerate the likelihood that a patient had PE. Other common findings were a lack of knowledge of the Wells score and time pressure influenced test choice.

We have highlighted gaps between evidence-based PE tests, the people who use the tests and environment where the tests are used. We found that test choices were influenced by the disease (fear associated with PE), the emergency department environment (time pressures and cognitive load), the test qualities (Wells 
score complexity), the physician (view of evidence-based medicine) and probability of the disease (evidence-based tests used more often when PE seems unlikely). The premise that an emergency physician will make testing decisions purely based on the numerical probability of disease seems unlikely to hold true in light of our findings. Probst and colleagues ${ }^{25}$ described the same issue in relation to ordering head CT scans for patients with head injuries in the emergency department, citing patient, system and physician factors influencing each decision. A 2015 qualitative study involving patients in the emergency department with minor head injuries reported that anxiety, time pressure, knowledge and patient factors also influenced the decision to order head CT. ${ }^{26}$ To effect changes in patient care, future tests should respond to a need identified by an emergency physician and should be designed specifically for the environement of the emergency department.

\section{Limitations}

There were limitations in our study design. We do not know whether those physicians who participated in our interviews differed from those who did not. We studied interview transcripts rather than real-time clinical observations. We did not record participant gender and therefore cannot comment on its effect. Our initial framework was derived from interviews performed at the Hamilton sites (which have a prominent thrombosis service). We did not identify practice differences among Hamilton and other sites; however, it is possible that the unique environment in Hamilton could have subconsciously shaped our framework. Our premise was that evidence-based PE testing was superior to alternative testing strategies, but we did not measure patient outcomes and cannot use our study findings to support this premise.

\section{Conclusion}

Analysis of structured interviews with emergency physicians provides a conceptual framework to explain how these physicians use tests for suspected PE, and why they choose the tests they use. These findings have important implications for future implementation in guidelines and the development of new PE tests.

\section{References}

1. Horlander KT, Mannino DM, Leeper KV. Pulmonary embolism mortality in the United States, 1979-1998: an analysis using multiple-cause mortality data. Arch Intern Med 2003;163:1711-7.

2. Swan D, Hitchen S, Klok FA, et al. The problem of under-diagnosis and overdiagnosis of pulmonary embolism. Thromb Res 2019;177:122-9.

3. Noble $\mathrm{S}$, Lewis $\mathrm{R}$, Whithers $\mathrm{J}$, et al. Long-term psychological consequences of symptomatic pulmonary embolism: a qualitative study. BMJ Open 2014;4: e004561.

4. Canadian Association of Emergency Physicians. Emergency medicine: ten things physicians and patients should question. Choosing Wisely Canada; updated 2019. Available: https://choosingwiselycanada.org/emergency-medicine/ (accessed 2020 June 1).

5. Avoid CT pulmonary angiography in emergency department patients with a low-pretest probability of pulmonary embolism and either a negative Pulmonary Embolism Rule-Out Criteria (PERC) or a negative D-dimer. Irving (TX): American College of Emergency Physicians; 2014. Available: www.choosingwisely.org/ clinician-lists/acep-ct-pulmonary-angiography-in-ed-patients/ (accessed 2020 June 1).
6. Raja AS, Greenberg JO, Qaseem A, et al.; Clinical Guidelines Committee of the American College of Physicians. Evaluation of patients with suspected acute pulmonary embolism: best practice advice from the Clinical Guidelines Committee of the American College of Physicians. Ann Intern Med 2015;163:701-11.

7. Kline JA, Mitchell AM, Kabrhel C, et al. Clinical criteria to prevent unnecessary diagnostic testing in emergency department patients with suspected pulmonary embolism. J Thromb Haemost 2004;2:1247-55.

8. Wells PS, Anderson DR, Rodger M, et al. Excluding pulmonary embolism at the bedside without diagnostic imaging: management of patients with suspected pulmonary embolism presenting to the emergency department by using a simple clinical model and D-dimer. Ann Intern Med 2001;135:98-107.

9. Righini M, Van Es J, Den Exter PL, et al. Age-adjusted D-dimer cutoff levels to rule out pulmonary embolism: the ADJUST-PE study. JAMA 2014;311:1117-24.

10. van der Hulle T, Cheung WY, Kooij S, et al. Simplified diagnostic management of suspected pulmonary embolism (the YEARS study): a prospective, multicentre, cohort study. Lancet 2017;390:289-97.

11. Kearon C, de Wit K, Parpia S, et al.; PEGeD Study Investigators. Diagnosis of pulmonary embolism with D-dimer adjusted to clinical probability. N Engl J Med 2019;381:2125-34.

12. Kearon C, Akl EA, Ornelas J, et al. Antithrombotic therapy for VTE disease: CHEST guideline and expert panel report. Chest 2016;149:315-52.

13. Konstantinides SV, Meyer G, Becattini C, et al.; The Task Force for the diagnosis and management of acute pulmonary embolism of the European Society of Cardiology (ESC). 2019 ESC Guidelines for the diagnosis and management of acute pulmonary embolism developed in collaboration with the European Respiratory Society (ERS): the Task Force for the diagnosis and management of acute pulmonary embolism of the European Society of Cardiology (ESC). Eur Respir J 2019;54:1901647.

14. Perelas A, Dimou A, Saenz A, et al. CT pulmonary angiography utilization in the emergency department: diagnostic yield and adherence to current guidelines. Am J Med Qual 2015;30:571-7.

15. Kline JA, Garrett JS, Sarmiento EJ, et al. Over-testing for suspected pulmonary embolism in American emergency departments: the continuing epidemic. Circ Cardiovasc Qual Outcomes 2020;13:e005753.

16. Simon E, Miake-Lye IM, Smith SW, et al. An evaluation of guideline-discordant ordering behavior for CT pulmonary angiography in the emergency department. J Am Coll Radiol 2019;16:1064-72.

17. Dhakal P, Iftikhar MH, Wang L, et al. Overutilisation of imaging studies for diagnosis of pulmonary embolism: are we following the guidelines? Postgrad Med J 2019;95:420-4

18. Andruchow JE, Grigat D, MCRae AD, et al. LO73: A randomized controlled trial of electronic clinical decision support to reduce unnecessary $\mathrm{CT}$ imaging for patients with suspected pulmonary embolism. CJEM 2018;20(Suppl 1):S32-3. doi: 10.1017/cem.2018.135.

19. Wang RC, Bent S, Weber E, et al. The impact of clinical decision rules on computed tomography use and yield for pulmonary embolism: a systematic review and meta-analysis. Ann Emerg Med 2016;67:693-701.e3.

20. Charmaz K. Constructing grounded theory: a practical guide through qualitative analysis. Thousand Oaks (CA): SAGE Publications; 2006.

21. Chan TM, Mercuri M, Turcotte M, et al. Making decisions in the era of the clinical decision rule: how emergency physicians use clinical decision rules. Acad Med 2020;95:1230-7.

22. Penaloza A, Verschuren F, Meyer G, et al. Comparison of the unstructured clinician gestalt, the Wells score, and the revised Geneva score to estimate pretest probability for suspected pulmonary embolism. Ann Emerg Med 2013;62:117-24.e2.

23. Gyftopoulos S, Smith SW, Simon E, et al. Qualitative study to understand ordering of CT angiography to diagnose pulmonary embolism in the emergency room setting. J Am Coll Radiol 2018;15:1276-84.

24. Westafer LM, Kunz A, Bugajska P, et al. Provider perspectives on the use of evidence-based risk stratification tools in the evaluation of pulmonary embolism: a qualitative study. Acad Emerg Med 2020;27:447-56.

25. Probst MA, Kanzaria HK, Schriger DL. A conceptual model of emergency physician decision making for head computed tomography in mild head injury. Am J Emerg Med 2014;32:645-50.

26. Melnick ER, Shafer K, Rodulfo N, et al. Understanding overuse of computed tomography for minor head injury in the emergency department: a triangulated qualitative study. Acad Emerg Med 2015;22:1474-83. 
Competing interests: Teresa Chan has received stipends from McMaster University and ALIEM LLC, and funding from the PSI Foundation outside the submitted work. No other competing interests were declared.

This article has been peer reviewed.

Affiliations: Faculty of Medicine (Zarabi, Varner) and Dalla Lana School of Public Health (Mercuri), University of Toronto, Toronto, Ont.; Faculty of Health Sciences (Chan, Mercuri, Kearon, de Wit), McMaster University, Hamilton, Ont.; Faculty of Medicine (Turcotte, Eagles), University of Ottawa, Ottawa, Ont.; Faculty of Medicine (Grusko), University of Manitoba, Winnipeg, Man.; Faculty of Medicine (Barbic), University of British Columbia, Vancouver, BC; Faculty of Medicine (Bridges), McGill University, Montréal, Que.; Chapel Hill School of Medicine (Houston), University of North Carolina, Chapel Hill, NC

Contributors: Kerstin de Wit conceived the study. Kerstin de Wit, Teresa Chan, Mathew Mercuri and Clive Kearon designed the study. Michelle Turcotte and Emily Grusko developed the interview methods. Analysis of data was conducted by Kerstin de Wit, Teresa Chan, Mathew Mercuri and Sahar Zarabi. Kerstin de Wit and Sahar Zarabi drafted the manuscript. All of the authors contributed to editing of the manuscript for important intellectual content, gave final approval of the version to be published and agreed to be accountable for all aspects of the work.
${ }^{*}$ Dr. Clive Kearon died on June 3, 2020, during preparation of this manuscript for publication.

Funding: Kerstin de Wit received the PSI Graham Farquharson Knowledge Translation Fellowship 2017-2020 and an Early Career Award from the Hamilton Health Sciences Foundation to fund the work of this study. Funding was also received from the McMaster Continuing Health Science Education Program Research and Innovation Fund.

Data sharing: Study coding is available on request to Kerstin de Wit (dewitk@mcmaster.ca).

Content licence: This is an Open Access article distributed in accordance with the terms of the Creative Commons Attribution (CC BY-NC-ND 4.0) licence, which permits use, distribution and reproduction in any medium, provided that the original publication is properly cited, the use is noncommercial (i.e., research or educational use), and no modifications or adaptations are made. See: https://creativecommons.org/licenses/ by-nc-nd/4.0/

Acknowledgement: The authors thank Natasha Clayton and the EMeRGE research program for their help to transcribe the interviews.

Accepted: Oct. 25, 2020

Correspondence to: Kerstin de Wit, dewitk@mcmaster.ca 